\title{
The Niguarda MEWS, a new and refined tool to determine criticality and instability in Internal Medicine Ward and Emergency Medicine Unit
}

\author{
Fabrizio Colombo, ${ }^{1}$ Lucia Taurino, ${ }^{2}$ Giulia Colombo, ${ }^{1}$ Massimo Amato, ${ }^{1}$ Salvatore Rizzo, ${ }^{1}$ Matteo Murolo, ${ }^{1}$ \\ Rita Facchetti, ${ }^{3}$ Ruggero Ruggeri ${ }^{1}$ \\ ${ }^{1}$ Internal Medicine Unit, Niguarda Ca' Granda Hospital, Milan; ${ }^{2}$ Emergency Medicine School, University of Florence, \\ Careggi Hospital, Florence; ${ }^{3}$ Department of Statistics, University of Milano Bicocca, Monza (MB), Italy
}

\begin{abstract}
This study compares the effect of the modified early warning score (MEWS) versus a new early warning system (Niguarda MEWS) for detecting instability and criticality in hospital medical departments. A retrospective observational study was conducted in the Internal Medicine ward of Niguarda Ca' Granda Hospital in Milan between November 2013 and October 2014. MEWS and Niguarda-MEWS were gathered using: systolic blood pressure, respiratory frequency, heart rate, temperature, level of consciousness, oxygen saturation, creatinine level, hematocrit level and age. In order to determine if the patient was critical or not the MEWS criticality cut-off value chosen was 3, while in the Niguarda MEWS it was 6 . The primary outcome was the correlation between the critical level of the two scores and in-hospital mortality. The secondary endpoint was the correlation between a specific disease and the two scores. In the study, 471 patients were included, using both the MEWS and the Niguarda MEWS score at admittance: $33.4 \%$ of patients turned out to be critically ill using the former, $40.98 \%$ when using the latter. Therefore, the specificity of scores was $70 \%$ for MEWS and $73 \%$ for Niguarda MEWS, the sensitivity $58 \%$ for MEWS and $63 \%$ for Niguarda MEWS, Niguarda MEWS area under the curve (AUC): 0.736, MEWS AUC: 0.670. For the secondary outcome, the new score is higher for genitourinary and respiratory diseases. Niguarda-MEWS could be an optimal tool to detect criticality and instability in order to address the patient to the right level of care.
\end{abstract}

\section{Introduction}

The evolution of the demand for healthcare and the development of a network of services led to a major review of the hospital role, which changed from being regarded as a privileged place of competent care with

Correspondence: Fabrizio Colombo, Internal Medicine Unit, Niguarda Ca' Granda Hospital, Piazza dell’Ospedale Maggiore 3, 20162 Milano, Italy.

Tel.: +39.02.644408.

E-mail: fabrizio.colombo@ospedaleniguarda.it

Key words: Modified early warning score; emergency; internal medicine; stratification of the risk; critical care.

Conflict of interest: the authors declare no potential conflict of interest.

Received for publication: 8 December 2016. Revision received: 28 March 2017.

Accepted for publication: 28 March 2017.

This work is licensed under a Creative Commons Attribution NonCommercial 4.0 License (CC BY-NC 4.0).

(C) Copyright F. Colombo et al., 2017

Licensee PAGEPress, Italy

Italian Journal of Medicine 2017; 11:310-317

doi:10.4081/itjm.2017.826 an exhaustive set of services to acute-care hospital, characterized by highly integrated technologies and expertise.

The more the hospital tends to define and guide its role in such direction, the more it becomes essential to establish the synergies which are necessary to an effective management of the treatment cycle and to organize the structure according to the intensity of care. ${ }^{1-3}$

This issue is extremely topical among the divisions of Internal Medicine.

The Internal Medicine operating units have been increasingly characterized for the treatment of acute patients. A progressive reduction in hospital beds, the increasing performance in day-hospital care, day-service and outpatient visits have concentrated patients suffering from serious diseases, both acute and acuteon-chronic, in medical wards. Furthermore, the increase in life expectancy has brought with it a kind of fragile patient, multi-pathological, at high risk of complications for comorbidity, and thus in need of intensive care. ${ }^{4,5}$

Intensity of care is related to many of these patients' instabilities and critical aspects; hospitalizing internal medicine patients in large wards without prior evaluation of these parameters and without prior risk stratification of rapid clinical deterioration may represent a non-optimal approach. 
There are limited data in literature about the availability of indicators and scores to attribute the level of intensity care in Internal Medicine. ${ }^{6,7}$

Several studies determined patient criticality and instability through the use of modified early warning score (MEWS), which is a simple, repeatable and bedside executable validated score that identifies critically-ill patients at risk of deterioration on the basis of simple parameters. ${ }^{8-14}$

Acute deterioration is often preceded by subtle changes in physiological parameters such as pulse, blood pressure, respiratory rate and level of consciousness.

Despite taking into consideration these parameters, MEWS does not exhaustively identify the criticality level of complex patients who are hospitalized in Internal Medicine wards.

Therefore, we extended the MEWS with some additional parameters, (i.e., $\mathrm{O}_{2}$ saturation, hematocrit, creatinine, and age) to introduce a new score (Niguarda MEWS).

The aim of this study was to compare the two scores (MEWS and Niguarda-MEWS) by evaluating their sensitivity, specificity and prognostic ability.

\section{Materials and Methods}

We enrolled patients admitted to the emergency room in the Internal Medicine ward of Niguarda hos- pital in the period from November 2013 to October 2014.

All patients with planned hospitalization were excluded from the analysis. In all patients with multiple admissions to the same department, only the last admission was taken into account.

The initial sample consisted of 490 patients.

The study was retrospective and employed the scores MEWS and Niguarda-MEWS (Table 1); all parameters were derived from data in the medical records at admission.

The MEWS score considers 5 parameters: systolic blood pressure, pulse rate, respiratory rate, temperature, and level of consciousness. ${ }^{8}$

The Niguarda-MEWS score takes also into account further parameters: $\mathrm{O}_{2}$ saturation, creatinine, hematocrit, and age.

A variable weight from 0 to 3 is assigned to each parameter in both scores.

The new score considers the same parameters as the MEWS with the addition of some refinements, whose data from literature have already validated as indicators of mortality: i) creatinine, whose range of values for scoring was extrapolated from APACHE II (acute physiologic assessment and chronic health evaluation II). ${ }^{15,16}$ According to this study, creatinine is to be scored 0 for values $<1.4 \mathrm{mg} / \mathrm{dL} ; 1$ for values between 1.5 and 2 $\mathrm{mg} / \mathrm{dL} ; 2$ for values between 2.1 and $2.9 \mathrm{mg} / \mathrm{dL}$; and 3 for values $>3 \mathrm{mg} / \mathrm{dL}$; ii) oxygen saturation by means of

Table 1. Modified early warning score (MEWS) and Niguarda MEWS.

\begin{tabular}{|c|c|c|c|c|c|c|c|c|}
\hline & \multicolumn{8}{|c|}{ MEWS } \\
\hline $\mathrm{SBP}(\mathrm{mmHg})$ & $<70$ & $71-80$ & $81-100$ & \multicolumn{2}{|c|}{$101-199$} & \multicolumn{3}{|c|}{$\geq 200$} \\
\hline Pulse rate (bpm) & - & $<40$ & $41-50$ & \multicolumn{2}{|c|}{$51-100$} & $101-110$ & $111-129$ & $\geq 130$ \\
\hline Respiratory rate (bpm) & - & $<9$ & - & \multicolumn{2}{|c|}{$9-14$} & $15-20$ & $21-29$ & $\geq 30$ \\
\hline Temperature $\left({ }^{\circ} \mathrm{C}\right)$ & - & $<35$ & - & \multicolumn{2}{|c|}{$35-38.4$} & - & $\geq 38.5$ & - \\
\hline \multirow[t]{3}{*}{ AVPU } & - & - & - & \multicolumn{2}{|c|}{ Alert } & Verbal & Pain & Unresponsive \\
\hline & \multicolumn{8}{|c|}{ Niguarda MEWS } \\
\hline & -1 & 3 & 2 & 1 & 0 & 1 & 2 & 3 \\
\hline $\mathrm{SBP}(\mathrm{mmHg})$ & - & $<70$ & $71-80$ & $81-100$ & $101-199$ & - & $\geq 200$ & - \\
\hline Pulse rate (bpm) & - & - & $<40$ & $41-50$ & $51-100$ & $101-110$ & $111-129$ & $\geq 130$ \\
\hline Respiratory rate (bpm) & - & - & $<9$ & - & $9-14$ & $15-20$ & $21-29$ & $\geq 30$ \\
\hline Temperature $\left({ }^{\circ} \mathrm{C}\right)$ & - & - & $<35$ & - & $35-38.4$ & - & $\geq 38.5$ & - \\
\hline AVPU & - & - & - & - & Alert & Verbal & Pain & Unresponsive \\
\hline Hematocrit (\%) & - & $<20$ & $20-29.9$ & & $30-45.9$ & $46-49.9$ & $50-59.9$ & $>60$ \\
\hline Creatinine $(\mathrm{mg} / \mathrm{dL})$ & - & - & - & - & - & $1.5-2$ & $2.1-2.9$ & $\geq 3$ \\
\hline Oxygen saturation (\%) & - & - & - & - & $100-95$ & $94-90$ & $89-85$ & $\leq 84$ \\
\hline Age (years) & $<56$ & - & - & - & $56-65$ & $66-74$ & $>75$ & - \\
\hline
\end{tabular}

MEWS, modified early warning score; SBP, systolic blood pressure; AVPU, level of consciousness. 
pulse oximeter, ${ }^{11,17,18}$ whose values were derived from Duckitt's study; ${ }^{17}$ iii) hematocrit, whose score is also derived from APACHE II. ${ }^{15,16}$

Basing on the scores assigned by APACHE II, normal ranges for hematocrit are between 30 and $45.9 \%$. One point was attributed when an increase of hematocrit between 46 and $49.9 \%$ was registered. Two points, which undoubtedly indicate a far more critical condition, were attributed in the presence of two possible conditions: either moderate-to-severe anemia (hematocrit 20-29.9\%) or moderate-to-severe hemoconcentration (hematocrit 50-59.9\%). Three points were attributed in the case of severe anemia or hemoconcentration, i.e. with values $<20 \%$ or $>60 \%$ respectively; iv) age: this parameter was chosen based on Smith's work. ${ }^{19}$ Patients aging between 56 and 65 years are not considered at risk. One point was taken off from the final score if the patient was under 55 years old.

\section{Statistical analysis}

In this work the Kruskal-Wallis test was used to compare the MEWS and the Niguarda-MEWS's scores between the diagnosis and the diagnosis-related group.

The $\chi^{2}$ test was employed to evaluate the association between diagnosis and death.

The discriminative power of the two scores was compared through the use of receiver operating characteristic (ROC) curves.

The study has been approved by the Institutional Reviewer Board of Niguarda Ca' Granda Hospital.

\section{Results}

Over the considered period, 490 patents were admitted to the Emergency Room of hospital Niguarda $\mathrm{Ca}$ ' Granda and discharged from departments of Internal Medicine (IA, IB, IC).

Eight patients were eliminated from the initial sample because they had been hospitalized twice and eleven patients were removed due to lack of data to determine the score. As a result, the total sample size was 471 patients. We considered 311 patients discharged in 2013 and 160 discharged in 2014.

Demographic characteristics of the studied patients are presented in Table 2.

The distributions of the two scores are presented in Figure 1.

We compared the two scores by assigning 0 to 3 points to each variable for both of them. Since MEWS considers five parameters, the scores range from 0 to 15, while Niguarda MEWS ranges from -1 to 21 .

As shown in the chart, the majority of patients tested through Niguarda MEWS scored between 3 and 8 .
Conversely, according to MEWS, most of the patients scored around 2 and 3.

The analysis through the ROC curves allowed to determine which of the two scores was more predictive of mortality; Niguarda MEWS emerged as more accurate than MEWS.

If we set the MEWS criticality cut-off value at 5 , which means that patients scoring $\geq 5$ should be admitted to intensive care units, $7.4 \%$ of patients turn out to be critically ill. On the other hand, if the criticality cutoff value is set at 3 , which is more pertinent to nonintensive medical units and to the concept of organizational appropriateness, $33.4 \%$ of patients turn out to be critically ill.

As already demonstrated by the study about care intensity and clinical complexity, ${ }^{20}$ considering criticality from scores $\geq 3$ would allow to more accurately predict the negative clinical evolution and thus to identify patients at risk of worsening.

In this study, the cut-off is set at 3 to maximize the specificity and sensitivity of the MEWS score, according to literature data. ${ }^{20,21}$ Conversely, referring to the Niguarda MEWS score, such value is set at 6 .

$40.98 \%$ of patients will be regarded as critically ill ( $7 \%$ more) by setting Niguarda-MEWS's criticality cut-off value $\geq 6$.

Through the evaluation of the cut-offs, we have

Table 2. Baseline demographic and clinical characteristics of the population sample $(n=471)$.

\begin{tabular}{lc}
\hline Characteristics & Data* \\
\hline Male prevalence & $228(48.41 \%)$ \\
\hline Age (years) & $77(67-84)$ \\
\hline Pulse rate (bpm) & $88(77-103)$ \\
\hline Respiratory rate (bpm) & $16(16-18)$ \\
\hline Systolic blood pressure (mmHg) & $140(115-155)$ \\
\hline Temperature $\left({ }^{\circ} \mathrm{C}\right)$ & $36(36-36.5)$ \\
\hline Serum creatinine (mg/dL) & $1.02(0.79-1.45)$ \\
\hline Hematocrit $(\%)$ & $36.8(31.5-41.3)$ \\
\hline Oxygen saturation $(\%)$ & $96(94-98)$ \\
\hline Hospitalization $($ days $)$ & $12(8-20)$ \\
\hline Expired & $57(12.1 \%)$ \\
\hline Others Departments & $48(10.19 \%)$ \\
\hline Intensive Care Unit & $1(0.21 \%)$ \\
\hline Emergency Medicine Department & $1(0.21 \%)$ \\
\hline Cardiac Coronary Unit & $1(0.21 \%)$ \\
\hline Sub-intensive Care Unit & $2(0.42 \%)$ \\
\hline Complications & $5(1.06 \%)$ \\
\hline
\end{tabular}

*Data are shown as median (interquartile range) or $\mathrm{N}(\%)$. 
thus assessed the sensitivity and specificity of both scores (Figure 2 and Table 3 ).

It is also very interesting to search for higher correlation between high values of both scores and a specific disease (Figures 3 and 4).

In this case, the new score is higher for genitourinary and respiratory diseases (median 6), followed by hematologic and cardiovascular diseases (5) and then by neurologic diseases (4).

On the contrary, MEWS has median 2 for respiratory and cardiovascular diseases.

It must be noted that the diseases which occur at older ages are those affecting: i) the central and pe- ripheral nervous system: median 82 ; ii) the cardiovascular system: median 82 ; iii) the genitourinary system: median 83.

Table 3. Sensitivity and specificity of cut-off values.

\begin{tabular}{lccc}
\hline & Cut-off & Sensitivity & Specificity \\
\hline MEWS & 3 & 0.578947 & 0.698068 \\
\hline Niguarda MEWS & 7 & 0.631579 & 0.724638 \\
\hline
\end{tabular}

MEWS, modified early warning score.

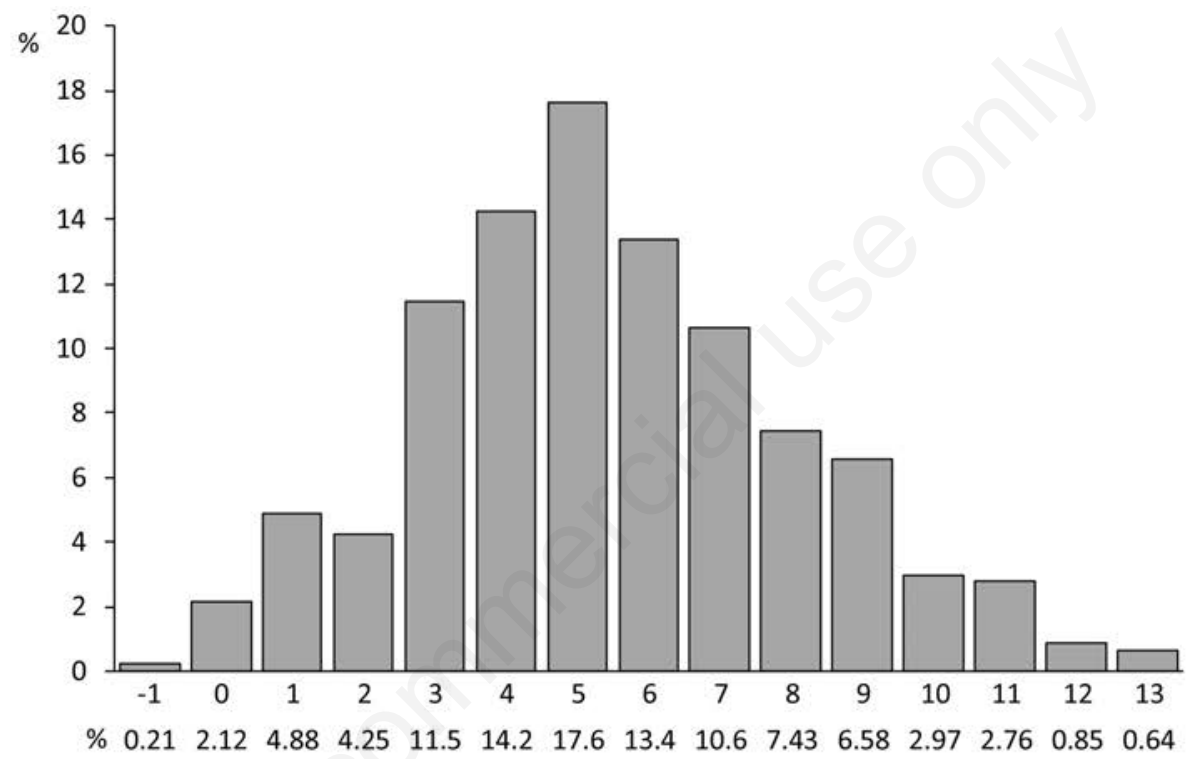

Niguarda MEWS

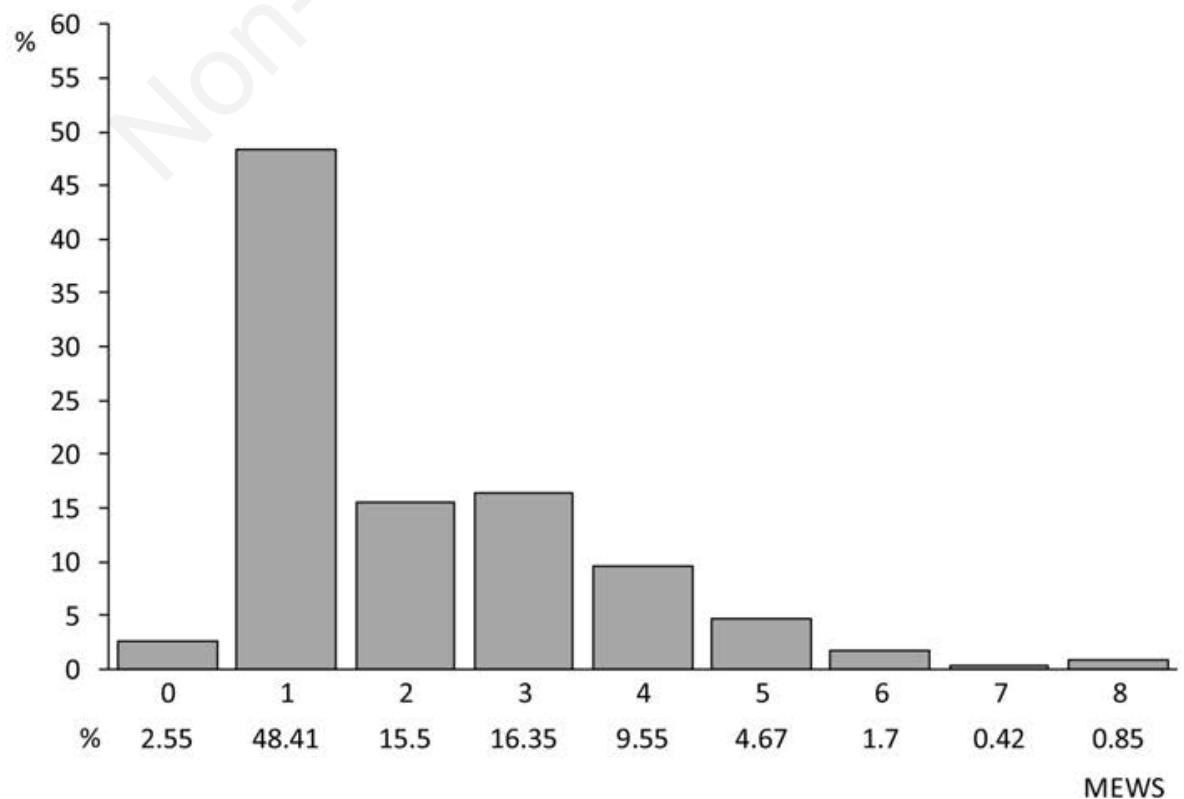

Figure 1. Distribution of modified early warning score (MEWS) (top panel) and Niguarda MEWS score (bottom panel). 
All these pathologies are correlated with higher scores and consequently with higher mortality, always highlighting the crucial role played by age in correlation with mortality, as can be easily understood.

\section{Discussion}

Our study is important because it confirms that hospitalizing patients without any preliminary stratification of risk may result in non-optimal treatment.

Unfortunately, it is still uncertain how many levels of care there are and, even worse, literature is scarce regarding the structuring of indicators to determine the level the patient should be assigned to.

Hospitals usually consider and provide for the following levels of care: i) level 1 (high intensity - critically ill patient); ii) level 2 (from acute to medium intensity).

There is also a level 3 (post-acute) which should be dealt with by local structures (Community Hospital, Nursing Home, Hospice).

In most cases, Internal Medicine departments operate at levels of care 1 and 2 (acute).

In particular, level 2 of Medicine appears of fundamental importance because it is where the majority of patients are admitted.

In fact, these patients do not require intensive care in the true sense of the word, but they need more care than what is regarded as normal for a traditional department.

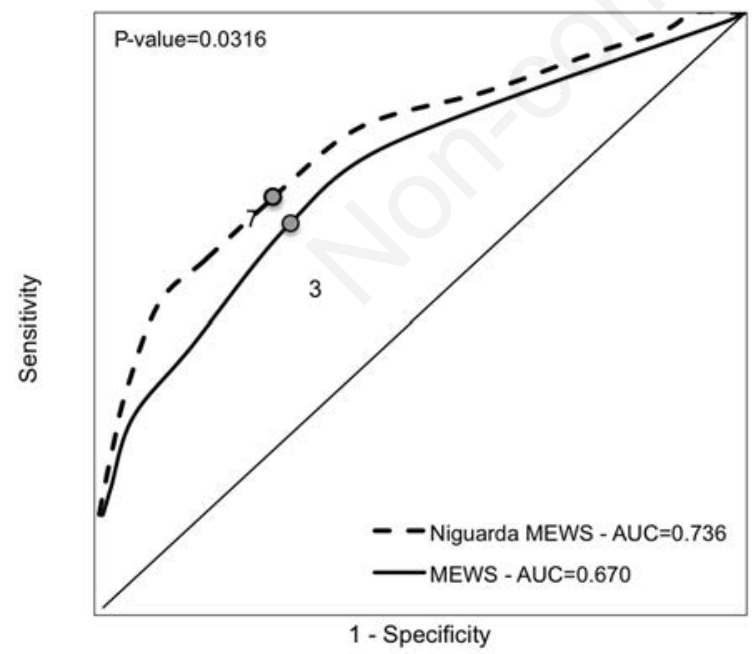

Figure 2. Receiver operating characteristic (ROC) curves for the predictive value of modified early warning score (MEWS) (solid line) and Niguarda MEWS (broken line) for intra-hospital death. P-value refers to comparison between the two areas under the curves (AUCs). Grey dots identify best cut-off values for each curve, which are the ones that optimize sensibility and specificity in the prediction.
A recent study based on data from Italian hospital discharge records has shown that Internal Medicine wards seem to have the most important role in the management of emergency hospitalizations: Internal Medicine wards receive $27 \%$ of the total emergency admissions and $83 \%$ of the admissions comes from Emergency Room. Furthermore, the results of the study support the phenomenon of the massive presence of elderly poly-pathological patients in Internal Medicine admissions (the highest number of hospitalizations in emergency is between 65 and over 85 years old) $;{ }^{22}$ in accord with these results, patients enrolled in our study are 77 (67-84) years old on average.

Despite not requiring intensive treatment, these patients may need a frequent assessment of vital signs and/or diagnostic and therapeutic interventions and/or focused nursing interventions.

Patient's admission to a ward, which is appropriate for its level of care, has to take into consideration the parameters of instability and criticality, conditions that may require a more frequent clinical monitoring of vital parameters, as well as more intensive therapies and nursing care.

Today there is no knowledge of precise indicators to determine the level of care and, in particular, literature is limited regarding the availability of scores to evaluate the complexity of hospitalized patients at admission..$^{23}$

Some studies employ MEWS to classify internal medicine patients at admission, ${ }^{24-26}$ but some others highlight the need for improvement and extension of parameters to better classify these patients. $23,27,28$

Much effort has recently been put by the UK National Health Service (NHS) into a standardized scoring system and, as a result, the national early warning score (NEWS) has been introduced. ${ }^{11}$ The NEWS, which adds $\mathrm{O}_{2}$ saturation to MEWS parameters, has shown its superiority in detecting clinical deterioration even if the positive impact on patient safety remains to be investigated.

Nevertheless, even with the addition of oxygen saturation NEWS score may not be able to recognize some internal medicine's acute diseases.

Additional parameters (age, hematocrit and creatinine in our method) may consent a more accurate stratification of clinical instability risk in Internal Medicine departments.

Old age and the complexity of patients with acute conditions have been indicated as major determinant for hospitalization through Emergency Room. ${ }^{22}$

The acute complex care model described by Pietrantonio et al. emphasizes the central role of the internist to ensure effective care of complex and poly-pathological patients hospitalized for acute illness. In this new model, high-dependency area in medical wards are devoted to unstable patients re- 
quiring assiduous monitoring of vital sign and/or complex therapies. ${ }^{29}$

Our study, in agreement with the work of Pietrantonio and Orlandini, confirms that the absence of a preliminary stratification of criticality, complexity and evaluation of a rapid clinical deterioration, can result in a non-optimal treatment especially for complex and poly-pathological patients who should be admitted to the high-dependency area in medical wards as provided in acute care complex model. ${ }^{29}$

\section{Study limitations}

A retrospective study with a small sample of patients does not allow an evaluation of the correlation between this new score and some complications such as hospitalization in Intensive and Sub-Intensive care and Cardiac Coronary Units.

Consequently, greater validation by means of a prospective study on a large scale is necessary, perhaps with the repetition of the new score during the days spent in the hospital. Instability and criticality are time-dependent and may change during hospital stay.

\section{Learning points}

The following learning points should be considered: i) MEWS, is a validated score that identifies critically-ill patients at risk of deterioration on the basis of simple parameters (systolic blood pressure, respiratory frequency, heart rate, temperature, level of consciousness); ii) Niguarda MEWS is a new early warning system that extended the MEWS with some additional parameters (i.e., $\mathrm{O}_{2}$ saturation, hematocrit, creatinine, and age); iii) the aim of this study was to compare the two scores (MEWS and NiguardaMEWS) ability in patients admitted to the emergency room in the Internal Medicine ward of Niguarda; iv) Niguarda-MEWS demonstrates higher sensitivity and specificity associated with greater prognostic and predictive ability compared to classic MEWS and may thus represent a more appropriate tool to detect criticality and instability in order to address the patient to the right level of care.

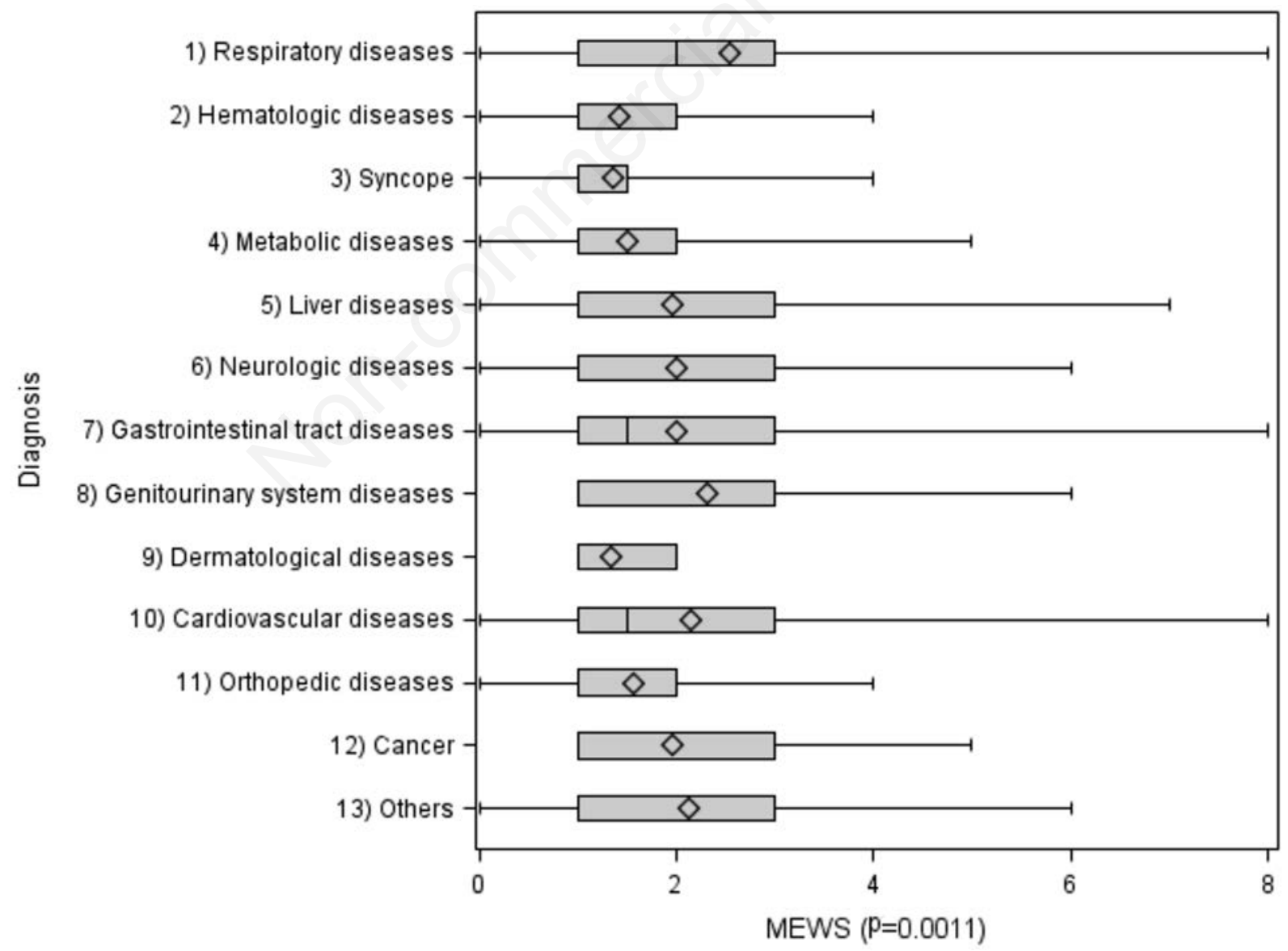

Figure 3. Distribution of modified early warning score (MEWS) according to diagnosis. Values are shown as median (vertical line within box), mean (diamond within box), $25^{\text {th }}$ and $75^{\text {th }}$ percentiles (boxes), and maximum-minimum ranges (vertical lines). P-value refers to comparison among scores. 


\section{Conclusions}

While confirming MEWS as a simple, quick repeatable and effective tool to detect criticalities at admittance, our study identifies a new score, which might be more useful for the evaluation of internal medicine patients. Both the scores can be measured at the bedside. As revealed by results from the analysis of the ROC curves, Niguarda-MEWS demonstrates higher sensitivity and specificity associated with greater prognostic and predictive ability and may thus represent a more appropriate tool in the medical sector for the identification of patients at risk of clinical deterioration and their consequent collocation, as well as a warning tool for operators. Both aspects are essential to outline a new model for intensity of care.

The Standard MEWS score explores five dimensions with a scoring range between 0 and 15 , while Niguarda MEWS score explores nine dimensions with a scoring range between 0 and 21 . In fact Niguarda MEWS maintains the previous characteristics of MEWS.
Standard MEWS is an assessment tool well validated by numerous studies about clinical criticality and instability, where five parameters were considered and patients were regarded as critically-ill for scores $\geq 5 .{ }^{8}$ If we set criticality cut-off value at 3 , as already done in other studies (which proved to be more realistic in medicine), we can identify $30 \%$ of criticallyill patients. ${ }^{20,21}$

Niguarda MEWS explores a greater number of dimensions and, with criticality cut-off value set at 7 , it also identifies $30 \%$ of critically-ill patients.

Consequently, for standard MEWS the criticality cut-off value is set at 3, while for Niguarda MEWS it is set at 7, thus allowing the identification of highly unstable patients. Hence the need to organize Internal Medicine Units by levels of care.

In the new score, criticality is correlated not only to cardio-respiratory diseases but also to hematologic and nephrological diseases, thus allowing the evaluation of other internal diseases.

Furthermore, it seems worth to stress that, since data are easy to collect in order to build the score, $\mathrm{Ni}$ -

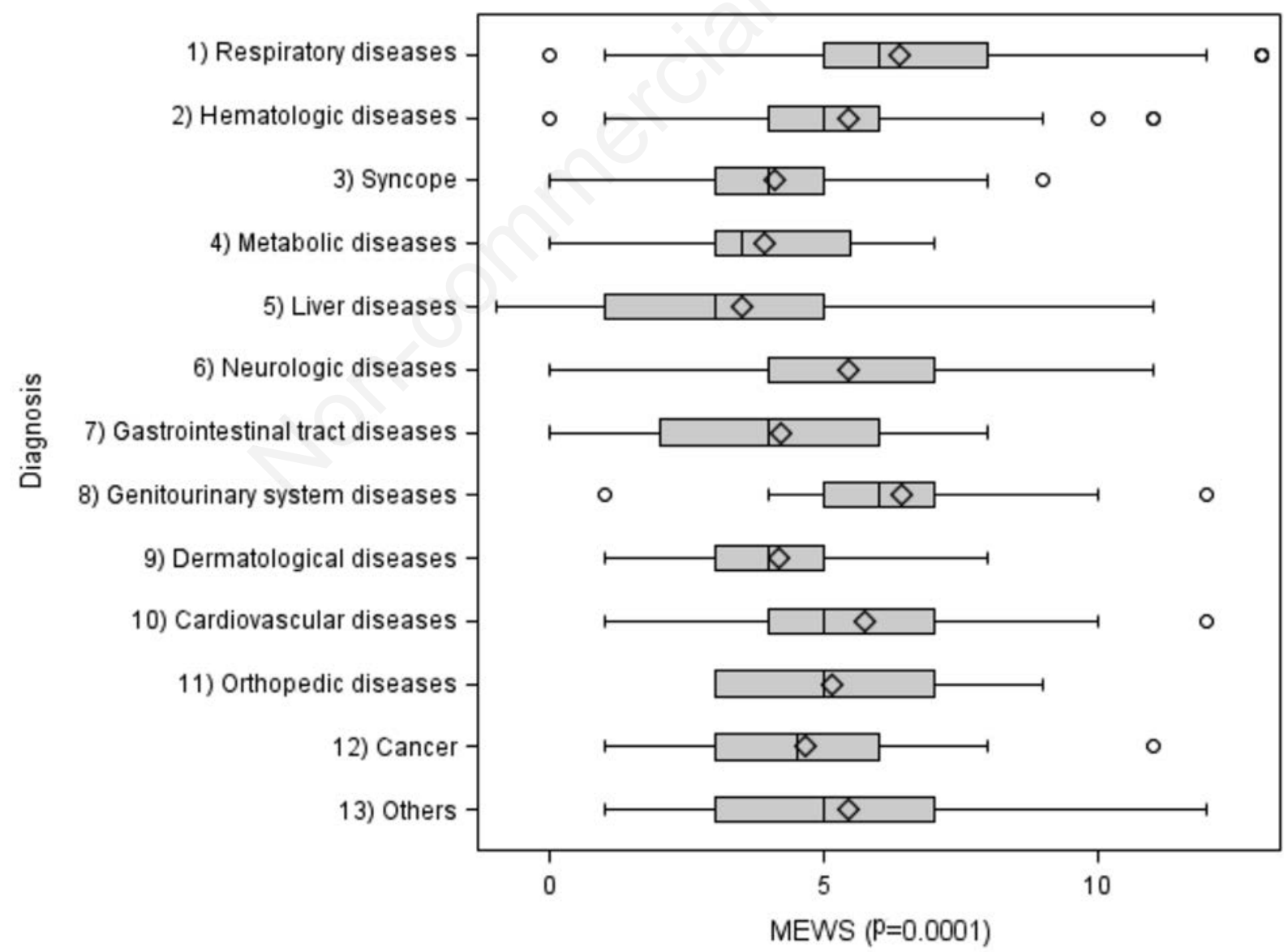

Figure 4. Distribution of Niguarda modified early warning score (MEWS) according to diagnosis. Values are shown as median (vertical line within box), mean (diamond within box), $25^{\text {th }}$ and $75^{\text {th }}$ percentiles (boxes), and maximum-minimum ranges (vertical lines). P-value refers to comparison among scores. 
guarda MEWS may be a valuable tool at the bedside, not only to classify the levels of care in medicine, but also to consider a reorganization of some departments such as Intensive Care Units and Emergency Medicine, where standard MEWS has already been employed, thanks to its immediacy and simplicity.

\section{References}

1. The Editors. Internal medicine and hospital organization based on treatment intensity: the position of FADOI Emilia Romagna. Ital J Med 2012;6:72-4.

2. Chesi G, Nardi R. Severity stratification of patients hospitalized on the Internal medicine ward: work in progress. Ital J Med 2013;7:231-3.

3. Briani S, Cortesi E. L'Ospedale per intensità di cure: aspetti teorici e problemi aperti. Ig. Sanità Pubbl 2007;63:577-86

4. Nardi R, Berti F, Greco A, et al. Complexity in hospital internal medicine departments: what are we talking about? Ital J Med 2013;7:142-55.

5. Nardi R, Fabbri T, Belmonte G, et al. Internal Medicine, complexity, evidence based medicine, almost "without evidences". Ital J Med 2009;3:191-200.

6. Bartolomei C, Cei M. The Allocation of patients in an intensity of care based Internal Medicine Ward: the ADOIT Tri-Co (Triage in Corridor) study. Ital J Med 2007;2:31-9.

7. Scotti E, Pietrantonio F. The hospital Internal Medicine specialist today: a literature review and strength, weakness, opportunity, threats (SWOT) analysis to develop a working proposal. Ital J Med 2013;7:278-86.

8. Subbe CP, Kruger M, Rutherford P, Gemmel L. Validation of a modified early warning score in medical admissions. Q J Med 2001;94:521-6.

9. Lee LL, Yeung KL, Lo WY, et al. Evaluation of a simplified therapeutic intervention scoring system (TISS28 ) and the modified early warning score (MEWS) in predicting physiological deterioration during inter-facility transport. Resuscitation 2008;76:47-51.

10. Hammond NE, Spooner AG, Barnett AG, et al. The effect of implementing a modified early warning scoring (MEWS) system on the adequacy of vital sign documentation. Aust Crit Care 2013;26:18-22.

11. Royal College of Physicians. National early warning score (NEWS) - Standardising the assesment of acuteillness severity in NHS. Report of a working party, July 2012. Available from: https://www.rcplondon.ac.uk/file/ 32/download?token $=5 \mathrm{NwjEyTq}$

12. Morgan RJM, Williams F, Wright MM. An early warning scoring system for detecting developing critical illness. Clin Intensive Care 1997;8:100.

13. Li YX, Ye HM. The prediction value of modified early warning score grade for death of the patients in intensive care unit. Chinese Crit Care Med 2008;20:419-21.

14. Fullerton JN, Price CL, Silvey NE, et al. Is the Modified early warning score (MEWS) superior to clinical judgement in detecting critical illness in the pre-hospital environment? Resuscitation 2012;83:557-62.
15. Wong DT, Knaus WA. Predicting outcome in critical care: the current status of the APACHE prognostic scoring system. Can J Anaesth 1991;38:374-83.

16. Knaus WA, Draper EA, Wagner DP, Zimmerman JE. APACHE II: a severity of disease classification system. Crit Care Med 1985;13:818-29.

17. Duckitt RW, Buxton-Thomas R, Walker J, et al. Worthing physiological scoring system: derivation and validation of a physiological early-warning system for medical admissions. An observational, population-based single-centre study. Br J Anaesth 2007;98:769-74.

18. Bayer O, Schwarzkopf D, Stumme C, et al. An early warning scoring system to identify septic patients in the prehospital setting: the PRESEP Score. Acad Emerg Med 2015;22:868-71.

19. Smith GB, Prytherch DR, Schmidt PE, et al. Should age be included as a component of track and trigger systems used to identify sick adult patients? Resuscitation 2008;78:109-15.

20. Bollini G, Colombo F. L'intensità assistenziale e la complessità clinica: un progetto di ricerca della Regione Lombardia. Milano: Era Futura; 2011. Available from: http://www.era-futura.com/editoria/

21. Pirneskoski J, Harjola VP, Jeskanen P, et al. Critically ill patients in emergency department may be characterized by low amplitude and high variability of amplitude of pulse photoplethysmography. Scand J Trauma Resusc Emerg Med 2013;21:48.

22. Pietrantonio F, Piasini L, Spandonaro F. Internal Medicine and emergency admissions: from a national Hospital Discharge Records (SDO) study to a regional analysis. Ital J Med 2016;10:157-67.

23. Alam N, Hobbelink EL, van Tienhoven AJ, et al. The impact of the use of Early Warning Scores (EWS) on patients ourcomes: a systematic review. Resuscitation 2014;85:587-94.

24. Cei M, Bartolomei C, Mumoli N. In-hospital mortality and morbidity of elderly medical patients can be predicted at admission by the modified early warning score: a prospective study. Int J Clin Pract 2009;63:591-5.

25. Burch VC, Tarr G, Morron C. Modified early warning score predicts the need for hospital admission and inhospital mortality. Emerg Med J 2008;25:674-8.

26. Kruisselbrink R, Kwizera A, Crowther M, et al. Modified early warning score (MEWS) identifies critical illness among ward patients in a resource restricted setting in Kampala, Uganda: a prospective observational study. PLoS One 2016 [Epub ahead of print].

27. Ludikhuize J, Borgert M, Binnekade J, et al. Standardized measurement of the modified early warning score results in enhanced implementation of a rapid response system: a quasi-experimental study. Resuscitation 2014;85:676-82.

28. Leung SC, Leung LP, Fan KL, Yip WL. Can prehospital modified early warning score identify non-trauma patients requiring life-saving intervention in the emergency department? Emerg Med Austral 2016;28:89-94.

29. Pietrantonio F, Orlandini F, Moriconi L, La Regina M. Acute complex care model: an organizational approach for the medical care of hospitalized acute complex patients. Eur J Intern Med 2015;26:759-65. 